\title{
Human-Robot Interaction during Walking with a Powered Compliant Knee Exoskeleton
}

\author{
Kristel Knaepen ${ }^{*}, \dagger$ \\ Friso Hagman ${ }^{*}, £$ \\ Pieter Beyl ${ }^{*}$, \\ Saartje Duerinck ${ }^{*}, ¥$ \\ Romain Meeusen ${ }^{*} \dagger$ \\ (*)Advanced Rehabilitation Technology and Science Research Group \\ (†)Department of Human Physiology \& Sports Medicine \\ (†)Robotics \& Multibody Mechanics Research Group, Department of Mechanical Engineering \\ (¥)Department of Experimental Anatomy \\ (£)Department of Biometrics and Biomechanics \\ Vrije Universiteit Brussel, Pleinlaan 2, B-1050 Brussels, Belgium \\ Email: kknaepen@vub.ac.be,pbeyl@vub.ac.be,sduerinc@vub.ac.be, fhagman@vub.ac.be, \\ rmeeusen@vub.ac.be
}

\begin{abstract}
Determinants of locomotor training involve taskspecificity, repeatability, variability, intensity and self-initiative. KNEXO, a unilateral knee exoskeleton has been developed to study the effects of compliant assistance during treadmill gait. Overall, walking within KNEXO, leads to asymmetric kinematics (Figure 1) and changes in naturally occurring muscle activity. Walking without KNEXO and with KNEXO in unassisted mode is difficult to compare as the device is unilateral and the pneumatic muscles give, although weight-compensated, a certain amount of inertia to the movement. Walking with KNEXO in high compliance resembles walking with KNEXO in unassisted mode. Overall, kinematic and EMG data show that the device has its shortcomings (unilateral, 1 DoF, 1 joint) when assisting healthy gait, yet it gives opportunities to study the effects of assistanceas-needed on gait biomechanics.
\end{abstract}

\section{Introduction}

Automated locomotor training allows for monitoring and assessment of gait training, long training sessions, high accuracy of desired gait patterns, and a reduced workload for physical therapists [6]. Yet, a monotonous repetition of the exact same gait pattern could result in learned disuse [14]. Determinants of gait training do not only involve task-specificity and repeatability, but also variability, intensity and self-initiative [9]. It seems to be primarily the effort of the patient that drives mechanisms of neuroplasticity [2]. A robotic gait orthosis should therefore be a compliant device that is able to interact with the subject to provide assistanceas-needed. To reduce the scale and complexity of the problem of compliant and safe human-robot interaction, a unilateral knee exoskeleton powered by pleated pneumatic muscles (KNEXO; at right leg) has been developed with built-in compliance [10, $11,12]$. This prototype was used to study the effects of compliant human-robot interaction on kinematics and muscle activity during gait in healthy subjects.

\section{Materials \& Methods}

\subsection{Subjects}

Ten healthy male subjects (age $27.4 \pm 6.1$ years, length $1.82 \pm 0.10 \mathrm{~m}$, weight $77.5 \pm 11.7 \mathrm{~kg}$ ) with no known neurological injuries or gait disorders gave their written informed consent and participated in this study. All experimental procedures were performed according to the standards set by the declaration of Helsinki for medical research involving human subjects and were part of a larger research project which has been approved by the medical ethics committee of the Vrije Universiteit Brussel.

\subsection{Instrumentation \& Data Acquisition}

\subsubsection{KNEXO (KNee EXOskeleton)}

The KNEXO device has previously been

This is an Open Access article distributed under the terms of the Creative Commons Attribution-Noncommercial License 3.0, which permits unrestricted use, distribution, and reproduction in any noncommercial medium, provided the original work is properly cited. 
described in detail by $[10,11]$. It is a unilateral powered knee exoskeleton (right knee) consisting of an upper and lower leg link connected by a hinge joint that rotates in the sagittal plane. The device is mounted over a treadmill and passively weightcompensated through a telescopic supportive arm to reduce asymmetrical loading of the subject. The 1 degree-of-freedom knee joint is powered by two Pleated Pneumatic Artificial Muscles (PPAMs [1, 4] in an antagonistic configuration. Subjects were strapped to the exoskeleton by four rigid, yet adaptable, leg cuffs around the upper and lower thigh and shank. The pelvis, hip and foot were not attached to the device, allowing free movement at those joints.

Depending on the walking mode, different control systems of KNEXO were used. Walking with KNEXO in unassisted mode was implemented by means of a torque controller. In order to mimic free treadmill walking while KNEXO is attached to the subjects' leg, the torque of the actuators is controlled towards zero so the subjects' gait is minimally impeded by the device. Therefore this mode was called zero-torque mode (ZT) [10].

For assisted walking, a proxy-based sliding mode controller (PSMC) was used with the difference in pressure $(\Delta p)$ instead of torque $(\Delta \tau)$ as the controller output. Unlike conventional trajectory controllers, a PSMC controller allows for suitable but less accurate tracking of the desired gait trajectory, while it answers in a slow and safe way to sudden perturbations from the desired trajectory (e.g. stumble)[8].

\subsubsection{Human Joint Kinematics}

A 3-dimensional motion analysis system, Vicon ${ }^{\circledR}$ 612-datastation by Vicon Motion Systems Ltd (Vicon-Oxford, UK), equipped with 7 MX F20 high speed infrared cameras and appropriate software, was used to track ankle, knee, hip and pelvis angular displacements and the position of the KNEXO relative to the subjects' limb. Using palpation methods, 53 technical makers with a diameter of 24 $\mathrm{mm}$ were placed on anatomical landmarks of the pelvis (7), anterior or lateral aspects of the left and right thigh and shank (16), anatomical landmarks of the feet (22) and on the upper and lower segment of the KNEXO (8).

\subsubsection{Electromyography}

To measure muscle electrical activity the Biomonitor ME6000 16 channel system from MegaWin by Mega Electronics Ltd (Kuopio, Finland) with surface electrodes Cleartrace
REF1700-03 by ConMed® Corporation (Utica, New York, USA) was used. The recording of data was done through MegaWin 3.0 b2 software at a sample rate of $1024 \mathrm{~Hz}$. Muscle activity of 5 muscles was recorded: right biceps femoris $(\mathrm{BF})$, vastus lateralis $(\mathrm{VL})$, rectus femoris (RF), tibialis anterior (TA), gastrocnemius medialis (GM). Electrode placement was done according to Seniam guidelines (www.seniam.org).

\subsubsection{Gait Phase Detection}

A force sensing resistor (FSR) placed on the sole of the subjects' right foot detects the moment of right heel strike and is used to synchronize both cycle initiation and duration of the subject and the exoskeleton on a step-by-step basis through a synchronization algorithm. Next to this, the FSR also synchronizes between the data captured by the exoskeleton, the motion analysis system and the EMG device. According to the heel strike detection signal, data are sectioned in gait cycles from $0 \%$ to $100 \%$ (i.e., right heel strike to right heel strike).

\subsection{Experimental Design}

On arrival in the lab subjects filled out an informed consent, an insurance certificate and a demographic questionnaire. Next KNEXO was fitted to the subjects' right leg (i.e., alignment between the axis of the human knee joint and the axis of the mechanical knee joint of the orthosis and fitting of the tailor-made cuffs). This was followed by a familiarization and warming-up period during which subjects walked on a treadmill with the device in unassisted mode, also zero-torque mode (ZT), during 5 to 10 minutes at a speed of $2.5 \mathrm{~km} / \mathrm{h}(0.7 \mathrm{~m} / \mathrm{s})$.

Before starting the actual experiment, measuring tools such as EMG electrodes and a foot contact sensor were attached to the subjects' right leg and foot and reflective markers to the subjects' pelvis, left and right leg and foot. To normalize the amplitude of EMG data, maximum voluntary contraction values (MVC) of the right BF, VL, RF, TA and GM muscle were recorded prior to the experiment. The actual experiment consisted of three consecutive blocks: (i) unassisted walking, (ii) assisted walking and (iii) assisted walking with resistance against the imposed trajectory. During the unassisted walking trials the ZT mode of KNEXO was assessed against regular treadmill walking.

We hypothesized that, at low speeds, walking with $\mathrm{KNEXO}$ in ZT would minimally affect the natural 
walking pattern of the subjects. Simultaneously, knee trajectories recorded during ZT were extracted, interpolated and averaged to a single reference trajectory (i.e., one gait cycle) that was used as a target trajectory during the assisted trials. All assisted walking trials started with 20 gait cycles in unassisted mode (ZT) before switching online to assisted mode. During the assisted walking trials different parameter settings of the control system (i.e., PSMC) were tested against each other. The PSMC parameters i.e., PID gains $\left(\mathrm{K}_{\mathrm{p}}, \mathrm{K}_{\mathrm{i}}, \mathrm{K}_{\mathrm{d}}\right)$, torque limit $\tau_{\text {lim }}$ and the sliding mode time constant $\lambda$, provide the ability to have gradations in the compliance of the system.

\subsection{Data Analysis}

The data (i.e., human kinematics, muscle activity, exoskeleton kinematics and kinetics) of 5 consecutive gait cycles of all walking were averaged based on right heel strike detection and time normalized $(0 \%$ $100 \%)$ to one averaged gait cycle with its' standard deviation ( $0 \%$ corresponds to right heel strike). Mean angular displacements $\left(^{\circ}\right)$ of the human ankle, knee, hip and pelvis and of the exoskeleton knee in the three spatial planes were calculated based on the relative motion of these segments to one another. The analysis of the EMG data was performed in Matlab by Mathworks Inc. (Natick, MA, USA). The raw EMG data were full rectified, normalized with respect to the MVC and time-averaged with a moving window of $100 \mathrm{~ms}$. For KNEXO the averaged achieved and target knee joint angles $\left(^{\circ}\right)$, torque $(\mathrm{Nm})$, power $(\mathrm{W})$ and work $(\mathrm{Nm})$ were calculated in Matlab by Mathworks Inc. (Natick, MA, USA).

\section{Results}

Averaged EMG data of 5 consecutive gait cycles of the right leg are shown in Figure 1. From the kinematic data, rotations in the sagittal plane will be discussed. Joint angles were averaged and time normalized to one gait cycle. Data are given for one healthy subject but are comparable for all 10 subjects.

When comparing walking without KNEXO and with KNEXO in ZF (unassisted), EMG data of the right leg showed increased activity of VL, RF and TA during initial contact, loading response and the end of swing, while the activity of BF during terminal stance and pre swing and the activity of GM during stance device is unilateral and the pneumatic muscles give, although KNEXO is weight-compensated, a certain amount of resistance to the movement. Walking with and initial swing are significantly reduced when walking with KNEXO in ZF (Figure 1).

Muscle activity during walking with KNEXO in $\mathrm{LC}$ no $\mathrm{R}$ and $\mathrm{HC}$ no $\mathrm{R}$ approaches muscle activity during walking with $\mathrm{KNEXO}$ in ZF.

Right knee excursion is reduced and right hip flexion increased when walking with KNEXO in ZF compared to walking without KNEXO (Figure 2).

When comparing walking with KNEXO in LC and $\mathrm{HC}$, joint kinematics are more pronounced when walking with KNEXO in LC. The subject is more forced into a desired gait pattern when walking in the LC mode.

\section{Discussion}

Increased activity of VL, RF and TA during initial contact, loading response and the end of swing could be in order to compensate the incomplete knee extension during swing, while the decreased activity of BF and GM are because of a limitation in the natural range of motion of the leg joints.

Right knee excursion is reduced and right hip flexion increased when walking with KNEXO in ZF compared to walking without KNEXO. This could be due to the inertia of the exoskeleton or to misalignments between the human joints and the robotic knee joint. Changes in kinematics of one joint also influence other joints.

When comparing walking with KNEXO in LC and $\mathrm{HC}$, joint kinematics are more pronounced when walking with KNEXO in LC. The subject is more forced into a desired gait pattern when walking in the LC mode.

The emphasis in this controller is put on its' compliant behavior rather than on the accuracy of the trajectory tracking. Together with the intrinsically compliant high power PPAM actuators, the PSMC control system is able to supply safe and adaptable guidance along a chosen trajectory. $[8,10,11]$.

\section{Conclusion}

Overall, walking within $\mathrm{KNEXO}$, which is a 1 DoF, 1 joint, unilateral device that limits the natural degrees of freedom of the leg, leads to asymmetric kinematics and changes in naturally occurring muscle activity. Walking without KNEXO and with KNEXO in unassisted mode is difficult to compare as the $\mathrm{KNEXO}$ in high compliance resembles walking with KNEXO in unassisted mode. 


\section{References}

[1] B. Verrelst, et al. Second generation pleated pneumatic artificial muscle and its robotic applications, Advanced Robotics, 20(7),783-805, 2006

[2] D. Reikensmeyer, et al. Some key problems for robot-assisted movement therapy research: a perspective from the university of california at Irvine, Proceedings of the 2007 IEEE International Conference on Rehabilitation Robotics, 1009-15, 2007

[3] D.A. Winter. The biomechanics and motor control of human gait: normal, elderly and pathological, University of Waterloo Press, 1991

[4] F. Daerden and D. Lefeber. The concept and design of pleated pneumatic arti cial muscles, International Journal of Fluid Power, 2(3), 41-50, 2001

[5] F. Dierick, et al. A force measuring treadmill in clinical gait analysis. Gait Posture, 20, 299-303, 2004

[6] G. Colombo, et al. Treadmill training of paraplegic patients using a robotic orthosis, $J$ Rehabil Res Devel, 37(6), 693-700, 2000

[7] J. Perry. The biomechanics and motor control of

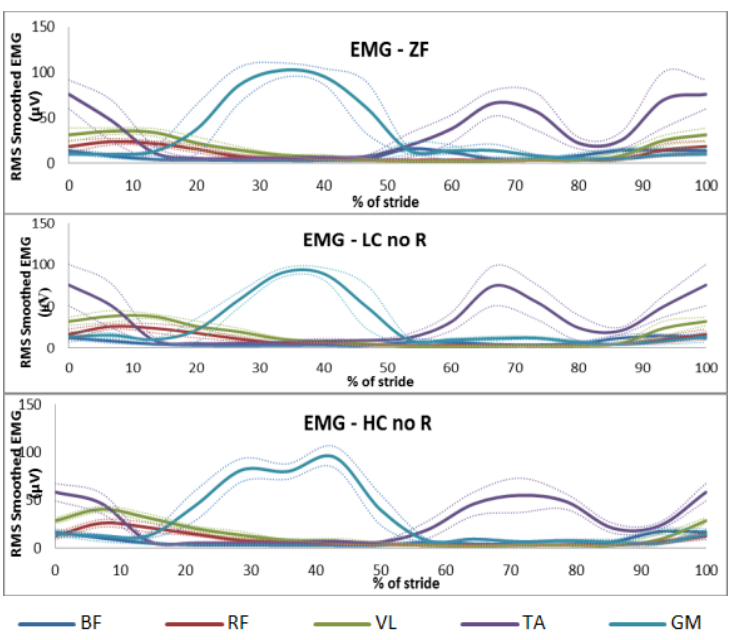

Figure 1: RMS smoothed, averaged EMG (+-stdev) data of 5 muscles of the right leg of a healthy subject during walking with KNEXO in ZF, LC no R, LC R, HC no $R$ and $H C R$ human gait: Normal, elderly and pathological, University of Waterloo Press, 1991

[8] M. Van Damme, et al. The safety of a robot actuated by pneumatic muscles: a case study. International Journal of Social Robotics, 2(3), 289-303, 2010

[9] M. Ziegler, et al. Why Variability Facilitates Spinal Learning, J NeuroSci, 30(32), 10720-26, 2010

[10] P. Beyl, et al. Safe and compliant guidance by a powered knee exoskeleton for robot-assisted rehabilitation of gait. Advanced Robotics, 25(5), 513-535, 2011

[11] P. Beyl, et al. Design and control of a lower limb exoskeleton for robot-assisted gait training, Applied Bionics and Biomechanics. 6(2), p. 229-43, 2009

[12] P. Beyl, et al. An Exoskeleton For Gait Rehabilitation: Prototype Design And Control Principle, Proceedings of the 2008 IEEE International Conference on Robotics and Automation, 2037-42, 2008

[13] R.B. Davis, et al. A gait analysis data collection and reduction technique, Hum Mov Sci, 10, 575-87, 1991

[14] V. Edgerton and R. Roy. Robotic training and spinal cord plasticity, Brain Res Bul, 78, 4-12, 2009

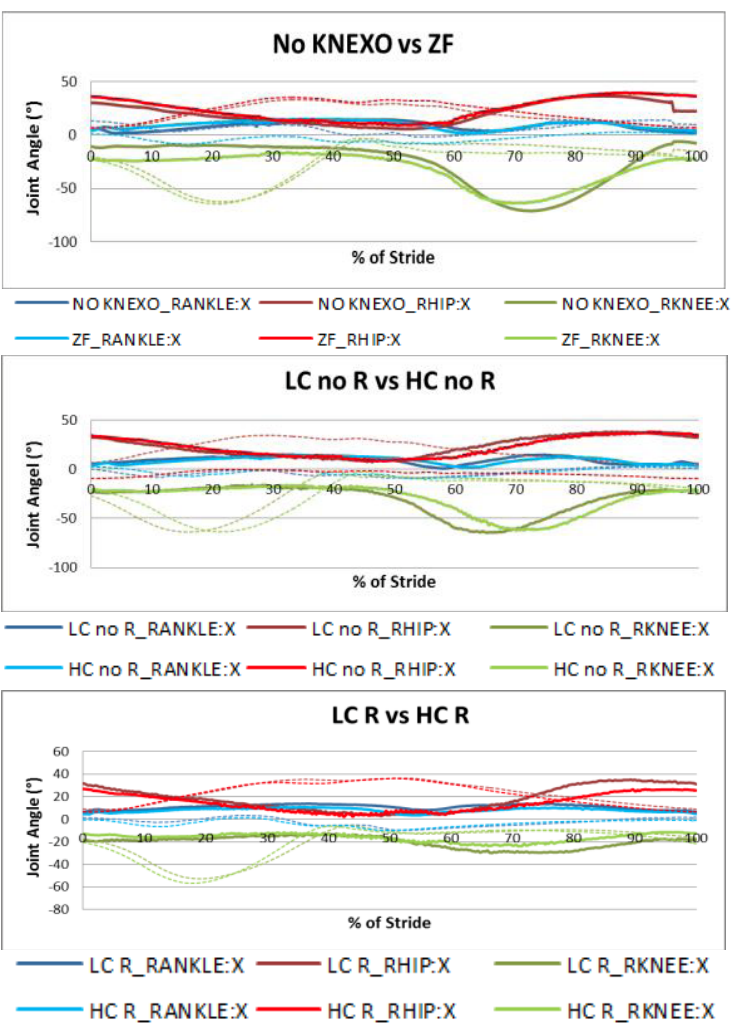

Figure 2: Comparison of the averaged and time normalized hip, knee and ankle joint angles of the left and right leg during walking without KNEXO and with KNEXO in ZF, LC no R, LC R, HC no R and HC R 